\title{
Mechanical Aspects of Biological Locomotion
}

\author{
A.E. Hosoi · E. Lauga
}

Published online: 17 November 2010

(C) The Author(s) 2010. This article is published with open access at Springerlink.com

Locomotion. The action or power of moving from one place to another; progressive motion of an animal. ... 1817 J. Evans Excurs. Windsor, etc. 451 The Oyster... was once thought to have no power of loco-motion, but it is now ascertained, that it can move from place to place. ...

\section{Oxford English Dictionary}

From oysters to bacteria to fish to flying snakes, locomotion is a pervasive feature of life. Many organisms rely on locomotion for survival-to escape predators, to find food or mates, or to migrate to suitable habitats. Animals have evolved a staggering array of appendages, structures, "motors," and kinematic strategies to generate propulsion. Today animal locomotion is a burgeoning field of research across many disciplines (biology of course, but also engineering, physics, and mathematics) and, as such, we have solicited a number of papers that examine motion through different fluid media. This resulting special issue contains not only novel research but also review papers that sum up the state-of-the-art of the field.

\footnotetext{
A.E. Hosoi $(\varangle)$

Department of Mechanical Engineering,

Massachusetts Institute of Technology,

Cambridge, MA 02139-4307, USA

e-mail: peko@mit.edu

E. Lauga

Department of Mechanical and Aerospace Engineering, University of California, San Diego, 9500 Gilman Drive, La Jolla, CA 92093-0411, USA

e-mail: elauga@ucsd.edu
}

The focus of this issue will be on propulsion though fluid media (e.g. air, water, and sand), as opposed to terrestrial locomotion which includes walking, running, hopping, etc. Such locomotion strategies can be roughly classified by the Reynolds number, Re, a dimensionless group that quantifies the relative magnitudes of viscous and inertial forces acted upon by the surrounding fluid [1]. The fifteen papers in this special issue have been organized by increasing values of Reynolds number; representative organisms from the included papers, and estimates of typical values of their Reynolds numbers are shown in Fig. 1.

We begin at one end of the spectrum with bacteria swimming at very low Reynolds numbers, typically $\operatorname{Re} \approx 10^{-5}-10^{-4}$, for which Yuan \& Berg study the rotary flagellar motor of $E$. coli under small loads. Moving on to eukaryotic cells, the next organisms to appear are spermatozoa where $\operatorname{Re} \approx 10^{-4}-10^{-3}$. In "How do sperm get to the egg? Bioengineering expertise needed!" Suarez outlines a number of open engineering challenges related to motility within the reproductive tract.

From there we move on to suspensions of swimmers at low Reynolds number and cooperative swimming strategies with three papers by Saintillan, Alexander \& Yeomans, and Pedley that address respectively the rheology of active suspensions, hydrodynamic interactions between active organisms in viscous fluids, and instabilities of swimming cells such as algae.

Intermediate Reynolds numbers $\left(\operatorname{Re} \approx 10^{-1}-1\right)$ are represented by the nematode $C$. elegans in a paper by Arratia, Shen \& Purohit, in which the authors investigate the effects of the viscosity of the surrounding medium on the kinematics of the organism. 


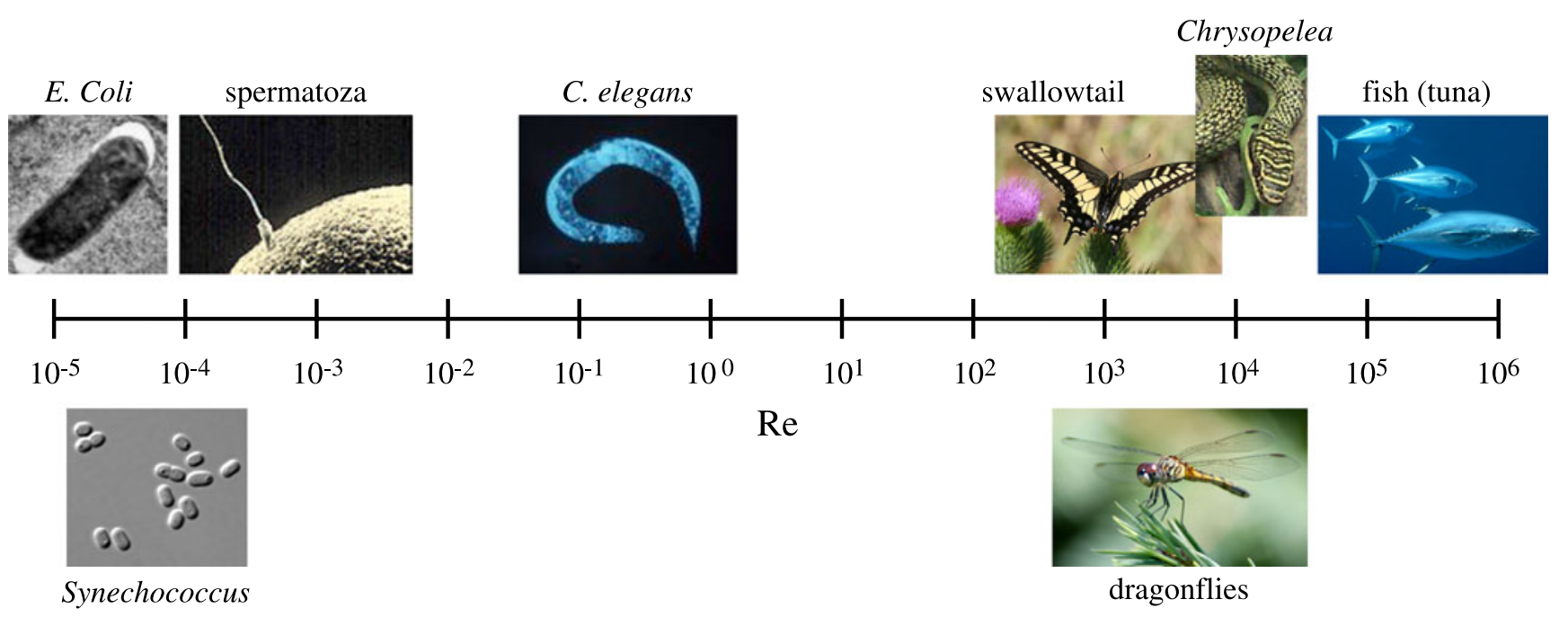

Fig. 1 Characteristic Reynolds numbers for various representative organisms appearing in, or relevant to, this special issue. Pictures reproduced courtesy of Wikimedia Commons (http://commons.wikimedia.org)

We then leave the realm of low Reynolds number locomotion and introduce moderate to high Re systems. We begin with insects which typically span Reynolds numbers ranging from $\mathrm{Re} \approx 100-10^{4}$. These system are illustrated in two studies: one on gliding swallowtail butterflies by Park, Bae, Lee, Jeon \& Choi, and one on the structure of dragonfly wings by Jongerius \& Lentink. This is followed by an investigation of the unusual locomotion strategy adopted by flying snakes as described by Miklasz, LaBarbera, Chen \& Socha.

The next four papers are motivated by fish locomotion, thus bridging the gap between moderate and high Reynolds number flows ( $\mathrm{Re} \approx 10^{6}$ and above). Eldredge presents a strategy to reconcile viscous and inviscid approaches to computing locomotion of deforming bodies, while Melli \& Rowley address "fishlike" locomotion in both the viscous and inviscid limits. These two studies lead us to a general description of flying and swimming at high Reynolds numbers by Eloy, Doare, Duchemin \& Schouveiler who present a unifying review of high Re models. Finally, we return to the question of collective and cooperative behavior, this time at the other end of the Reynolds number spectrum in a paper by Kanso on the advantages of flapping out of phase with nearby swimmers.

The two concluding papers fall outside the realm of our Reynolds number classification scheme as, owing to complexity of surrounding medium in these two studies, the dynamics cannot be characterized by a single dimensionless number. Both of these investigations address systems that move through, or on, a granular medium. The first by Dorgan, presents a review of different burrowing and crawling strategies through complex media; the second, by Li, Umbanhowar, Komsuoglu \& Goldman offers a study of locomotion on a dry granular medium.

As we edited this special issue over the last year, a few common themes emerged. One of the unique aspects of locomotion through a fluid environment is that the medium has the potential, through both pressure and viscous stresses, to transmit forces between organisms. This can lead to a number of interesting swimming strategies that exploit the presence of neighboring swimmers, as well as collective behavior among large numbers of individuals. This type of cooperative behavior is addressed in several papers in this issue, at both ends of the Reynolds number spectrum.

Another theme that runs through a number of locomotion studies is the concept of optimization. The idea that one can use optimization to address biological shape and function is, at best, controversial. The argument against optimization is not that natural selection fails to (eventually) optimize biological organisms, rather that biological systems are too complex to be amenable to such analyses. Very often many objectives exist simultaneously requiring multiple (often unknown) cost functions, with unknown weights. In addition, the parameter space is vast with many interacting variables-geometry, material properties, kinematics. The environment is constantly changing hence evolving organisms are "chasing a moving target" making optimization studies challenging. Nevertheless, a number of the contributors to this special issue present studies that have made advances on this front, in particular, studies pertaining to optimal kinematics for given morphologies. 
We were also interested to note the variety of research approaches undertaken. The range of tools developed in the papers gathered here range from scaling and asymptotic theories to full numerical simulations to experimental investigations. Sometimes similar questions are addressed with different points of views by different authors, for example the discrete approach to collective cell behavior developed by Alexander \& Yeomans versus the continuum description of Pedley.

A final observation based on this collection of papers is that the primary focus of locomotion research seems to no longer be that of body kinematics, in the spirit of the pioneering studies of Gray [2], but instead new questions dealing with material properties, organisms energetics, and locomotion efficiency are taking center stage. We are excited by these new directions and hope that this special issue, with its range of organisms, approaches, and regimes, will help galvanize the next generation of locomotion research.

Open Access This article is distributed under the terms of the Creative Commons Attribution Noncommercial License which permits any noncommercial use, distribution, and reproduction in any medium, provided the original author(s) and source are credited.

\section{References}

1. Batchelor, G.K.: An Introduction to Fluid Dynamics. Cambridge University Press, Cambridge, UK (1967)

2. Gray, J.: Animal Locomotion. Norton, London, UK (1968) 